\title{
Reaction-Diffusion Modelling of Interferon Distribution in Secondary Lymphoid Organs
}

\author{
G. Bocharov ${ }^{1 * \dagger}$, A. Danilov ${ }^{1 * \dagger}$, Yu. Vassilevski ${ }^{1}$, \\ G.I. Marchuk ${ }^{1}$, V.A. Chereshnev ${ }^{2}$ and B. Ludewig ${ }^{3}$ \\ ${ }^{1}$ Institute of Numerical Mathematics, Russian Academy of Sciences, Moscow, Russia \\ ${ }^{2}$ Institute of Immunology and Physiology, Ural Branch of the Russian Academy of Sciences, \\ Ekaterinburg, Russia \\ ${ }^{3}$ Institute of Immunobiology, Cantonal Hospital of St. Gallen, St. Gallen, Switzerland
}

\begin{abstract}
This paper proposes a quantitative model of the reaction-diffusion type to examine the distribution of interferon- $\alpha$ (IFN $\alpha$ ) in a lymph node (LN). The numerical treatment of the model is based on using an original unstructured mesh generation software Ani3D and nonlinear finite volume method for diffusion equations. The study results in suggestion that due to the variations in hydraulic conductivity of various zones of the secondary lymphoid organs the spatial stationary distribution of IFN $\alpha$ is essentially heterogeneous across the organs. Highly protected domains such as sinuses, conduits, co-exist with the regions in which where the stationary concentration of IFN $\alpha$ is lower by about 100 -fold. This is the first study where the spatial distribution of soluble immune factors in secondary lymphoid organs is modelled for a realistic three-dimensional geometry.
\end{abstract}

Key words: mathematical model, immune response, reaction-diffusion equation, 3D distribution of interferon- $\alpha$

AMS subject classification: $92 \mathrm{~B} 05,92 \mathrm{C} 42$

\footnotetext{
*Corresponding authors. E-mails: bocharov@inm.ras.ru, a.a.danilov@gmail.com

$\dagger$ First authors contributed equally to this work
} 


\section{Introduction}

Many virus infections such as influenza, SARS-coronavirus, hepatitis C, HIV represent major threats to human health. Protection against the virus infections is a main function of the immune system. Human immune system is composed of compartmentalized populations of different types of cells and soluble factors. Their functioning and dynamics are governed by a complex and tightly regulated set of physical, biochemical and biological processes occurring in space and time. The immune system responses are associated with the activation of various subsets of specialized cells, including antigen-presenting cells (APCs) and antigen-specific lymphocytes which proliferate, differentiate, mature and secrete a broad range of soluble factors (cytokines, lymphokines, antibodies etc.) and finally die. The initial innate immune responses to cytopathic viruses have to restrict the virus spread before the adaptive virus-specific immune responses develop. In addition, they have to protect the antigen-presenting cells to ensure a sustained stimulation of the adaptive immune responses by the macrophages and dendritic cells. In experimental cytopathic coronavirus infection the early APCs protection of the APCs is mediated by the antiviral interferon- $\alpha$ (IFN $\alpha$ ) response of plasmacytoid dendritic cells (pDCs) [7]. Importantly, it has been demonstrated that activated pDCs strongly suppress HIV replication in $C D 4^{+} \mathrm{T}$ cells via IFN $\alpha$-related mechanism [23].

Secondary lymphoid organs (SLO) are tissues where the induction of innate and adaptive immune responses takes place. SLO microenvironment provides the structural basis for the induction of T- and B-cell responses. The efficacy of immune responses relies on a productive interaction between antigen-presenting cells and lymphocytes. APCs protection by interferon requires that the in situ IFN $\alpha$ concentration should exceeds a certain threshold [7]. The interferon produced by pDCs is spatially distributed via convection and diffusion processes to various SLO compartments. The microanatomy of a paradigmatic SLO can be essentially reduced to three zones: the subcapsular zone, which is an APC-rich area, the B-cell activation zone (B-cell follicle) and T-cell activation zone [12]. Although direct measurements of the hydraulic conductivity within the T-cell zone are missing, it is considered that both diffusion and convection are extremely low [16]. Therefore, the spatial distribution of IFN in SLO deserves further investigation.

The immune system is very complex, and the quantitative predictive understanding of its regulation requires integration of experiments aimed at gathering data at various levels of the immune system organization and the mathematical theory based upon the powerful methods of systems analysis and computational modelling technologies [1, 5, 6, 19,21,24]. Our objective is to develop a three-dimensional (3D) geometric model of a paradigmatic secondary lymphoid organ to examine interferon spatial distribution using a reaction-diffusion equation. Few previous publications on the spatio-temporal dynamics of immune responses mainly focused on a systemic recirculation of lymphocytes between various organs of the immune system ignoring the SLO microanatomy [10, 28, 29]. There were a pioneer work of Mohler et al., [22] and a recent study of Baldazzi et al. [2], where the lymph node spatial compartmentalization was considered to describe the lymphocyte traffic. However, all the above mentioned studies do not consider the spatial distribution of immune responses soluble factors for realistic 3D geometry of the SLO, that should allow to take into account the variations in hydraulic conductivity across the different SLO zones. In this paper we propose a quantitative model of reaction-diffusion type to examine the distribution of 
IFN $\alpha$ in a lymph node (LN). The numerical treatment of the model is based on using the original unstructured mesh generation software Ani3D [8] and nonlinear finite volume method for diffusion equations [9].

In Section 2 we present an interferon reaction model which quantifies the dynamics of IFN $\alpha$ response applying a compartmental approach. It is used to specify the parameters for reactiondiffusion model formulated in Section 3. The 3D geometry of a paradigmatic LN is described in Section 4 together with the approximating tetrahedral mesh based model. The details of the diffusion equation numerical solution and the results for various biologically relevant scenarios of parameter values are described in Section 5. Section 6 contains concluding remarks.

\section{The compartmental model of IFN response}

The first step in the computational modelling of the IFN $\alpha$ spatio-temporal dynamics is to quantify the kinetic parameters of the interferon response using experimental data. To this end we used high-resolution data coming from the well-described mouse hepatitis virus infection and the set of delay-differential equations describing the interaction between the virus, plasmacytoid dendritic cells and macrophages. The model is formulated using the systems engineering approach and considers a temporal dynamics of interferon $I(t)$ and uninfected/infected pDCs and macrophages $C^{p D C}(t), C^{M \varphi}(t), C_{V}^{p D C}(t), C_{V}^{M \varphi}(t)$ in spleen, as well as a systemic dynamics of the virus in spleen, blood and liver $V_{S}(t), V_{B}(t), V_{L}(t)$. The corresponding equations are formulated by means of well-established approach $[5,19]$ (we refer to [7] for further details):

$$
\begin{aligned}
\frac{d I}{d t}(t)= & \rho_{I}^{p D C} C_{V}^{p D C}\left(t-\tau_{I}^{p D C}\right)+\rho_{I}^{M \varphi} C_{V}^{M \varphi}\left(t-\tau_{I}^{M \varphi}\right)-d_{I} I(t), \\
\frac{d C_{V}^{p D C}}{d t}(t)= & \sigma_{V}^{p D C} V_{S}(t) C^{p D C}(t)-d_{0 C V}^{p D C} C_{V}^{p D C}(t), \\
\frac{d C_{V}^{M \varphi}}{d t}(t)= & \sigma_{V}^{M \varphi} V_{S}(t) C^{M \varphi}(t)-d_{0 C V}^{M \varphi} C_{V}^{M \varphi}(t), \\
\frac{d C^{p D C}}{d t}(t)= & -\sigma_{V}^{p D C} V_{S}(t) C^{p D C}(t)+d_{0 C}^{p D C}\left(C_{0}^{p D C}-C^{p D C}(t)\right), \\
\frac{d C^{M \varphi}}{d t}(t)= & -\sigma_{V}^{M \varphi} V_{S}(t) C^{M \varphi}(t)+d_{0 C}^{M \varphi}\left(C_{0}^{M \varphi}-C^{M \varphi}(t)\right), \\
\frac{d V_{S}}{d t}(t)= & \frac{\rho_{V}^{p D C}}{1+I(t) / \theta_{p D C}} C_{V}^{p D C}\left(t-\tau_{V}^{p D C}\right)+\frac{\rho_{V}^{M \varphi}}{1+I(t) / \theta_{M \varphi}} C_{V}^{M \varphi}\left(t-\tau_{V}^{M \varphi}\right) \\
& -\left(\sigma_{V}^{p D C} C^{p D C}(t)+\sigma_{V}^{M \varphi} C^{M \varphi}(t)\right) V_{S}(t)-d_{V} V_{S}(t) \\
& -\mu_{S B} V_{S}(t)+\mu_{B S} V_{B}(t) \frac{Q_{B}}{Q_{S}},
\end{aligned}
$$




$$
\begin{aligned}
\frac{d V_{B}}{d t}(t) & =\mu_{S B} V_{S}(t) \frac{Q_{S}}{Q_{B}}+\mu_{L B} V_{L}(t) \frac{Q_{L}}{Q_{B}}-\left(\mu_{B S}+\mu_{B L}+\mu_{B O}\right) V_{B}(t), \\
\frac{d V_{L}}{d t}(t) & =\beta_{L} V_{L}(t)\left(1-V_{L}(t) / K_{L}\right)-\mu_{L B} V_{L}(t)+\mu_{B L} V_{B}(t) \frac{Q_{B}}{Q_{L}}
\end{aligned}
$$

The model was used to estimate IFN $\alpha$ concentration of which is required to inhibit by two fold virus production by the infected cells. It appears that the $\mathrm{pDC}$ and $\mathrm{M} \varphi$ differ with respect to the protective effect of the interferon, so that the $50 \%$ reduction of the threshold concentrations are about $0.046 \mathrm{pg} / \mathrm{mm}^{3}$ and $0.001 \mathrm{pg} / \mathrm{mm}^{3}$, respectively.
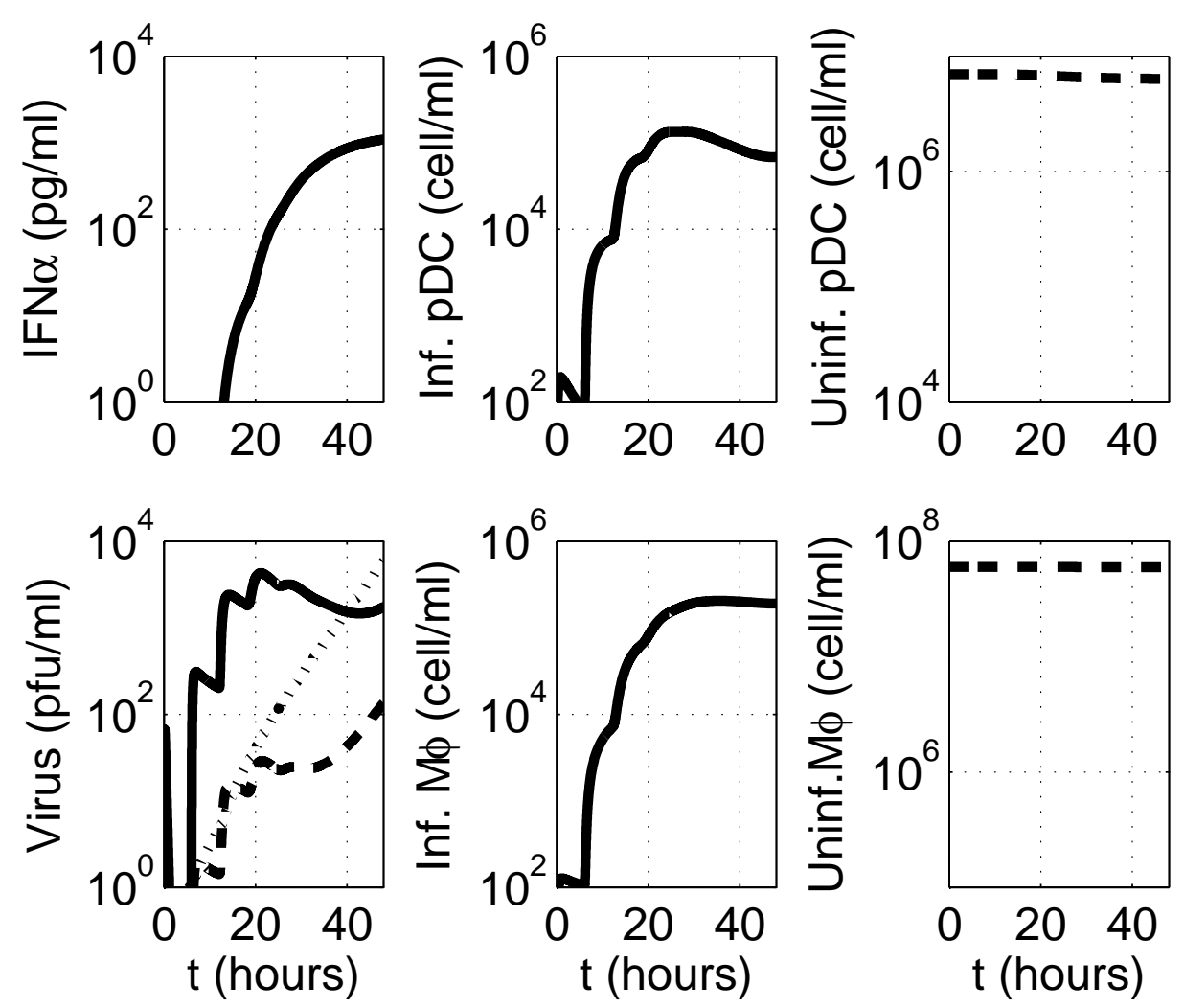

Figure 1: The solution of the compartmental model describing the kinetics of interferon response (upper left panel) and the population dynamics of virus (lower left panel) in spleen (solid line), liver (dotted line) and blood (dashed line), respectively, infected/uninfected pDCs (upper middle and right panels), and infected/uninfected macrophages (lower middle and right panels). The parameters of the interferon response were estimated from in vitro and in vivo (spleen) data. The compartmental approach assumes an instantaneous mixing of IFN $\alpha$ implying that the concentration across the SLO (spleen) is uniform. 


\section{Reaction-diffusion model of IFN dynamics}

The above set of delay-differential equations modelling the interferon reaction was developed following a compartmental approach, i.e. assuming the secondary lymphoid organ (spleen) in which the reaction takes place to be spatially homogeneous with instantaneous mixing. However, the secondary lymphoid organs have an elaborated architecture. The details of their structural organization allow to suggest that an intensity of the transport processes, in particular, the diffusion, mediating the spread of soluble factors, in particular, IFN $\alpha$, differs essentially depending on the location in the specific anatomical SLO compartments [16]. To examine the diffusion effect on the spatial distribution of IFN $\alpha$ we consider the concentration of the interferon $I$ at time $t$ to be also dependent on position $\mathrm{x} \in \Omega \subset R^{3}$, where $\Omega$ represents the spatial region occupied by the lymphoid organ. In the case of a paradigmatic SLO it consists of a number $(N)$ of non-overlapping subdomains $\Omega_{i}: \Omega=\bigcup_{i=1}^{N} \Omega_{i}$. The spatio temporal dependence of the variable $I(\mathbf{x}, t)$ is treated as a continuum field of concentration evolving according to the reaction-diffusion equation with a source term:

$$
\frac{\partial I}{\partial t}(\mathbf{x}, t)=\nabla \cdot(\mathbb{D} \nabla I(\mathbf{x}, t))-d_{I} I(\mathbf{x}, t)+\sum_{l=1}^{L} F_{l}(\mathbf{x}) .
$$

Here $\mathbb{D}$ stands for the diffusion mass transfer tensor which is assumed to be a scalar constant coefficient depending on the subdomain $\left(\mathbb{D}=D_{i} \cdot \mathbb{I}, i=1, \ldots, N\right)$ since diffusion is considered to be isotropic. Interferon degradation is described by the term $-d_{I} I(\mathbf{x}, t)$. IFN $\alpha$ secretion by different type activated cells located at some positions $\left(\mathbf{x}_{k}^{m}\right)_{k=1, K_{l}}^{l=1, L}$ is represented by the source term. It is the sum over Dirac delta functions $F_{l}(\mathbf{x})=\sum_{k=1}^{K_{l}} \rho^{(l)} \delta\left(\mathbf{x}-\mathbf{x}_{k}^{(l)}\right)$ with $\rho^{(l)}$ representing the per capita cell type specific secretion rate. Due to the singularity of $F_{l}(\cdot)$ the equations are understood in the weak sense [20].

The production-degradation parameters were estimated based on the actual data using the compartmental model in [7]. In particular, the production rates of IFN $\alpha$ by activated pDC and macrophage are $\rho^{p D C}=4.4 \times 10^{-4} \mathrm{pg} / \mathrm{h} /$ cell and $\rho^{M \varphi}=10^{-6} \mathrm{pg} / \mathrm{h} / \mathrm{cell}$, respectively. The estimated decay rate of IFN $\alpha$ in a cell-free medium is $d_{I}=0.012 \mathrm{~h}^{-1}$. The last value does not consider the consumption of interferon and can be increased by up to ten fold to account for the internalization of free IFN $\alpha$ by various cells in SLO.

The diffusion characteristics of the subdomains representing various compartments of a paradigmatic secondary lymphoid organ and corresponding boundary conditions are discussed in the next section.

\section{3D approximation of a paradigmatic lymph node}

Secondary lymphoid organs have highly elaborate structure and organization to facilitate the interaction between the immune cells and the lymph-borne pathogens derived from distant tissues. 
Following a building-block approach a paradigmatic lymph node synthesis is presented in [12]. Functionally, the lymph node (domain $\Omega$ ) consists of three major subdomains:

- an outer antigen-sampling zone (subcapsular sinus, trabecular sinuses, conduit tubes), referred to as subdomain $\Omega_{1}$,

- B-cell follicles which make subdomain $\Omega_{2}$,

- T-cell zone (cortex and paracortex) denoted as subdomain $\Omega_{3}$.

A paradigmatic LN schematic view is presented in Fig. 1. The subcapsular sinus in LN contains aggregates of macrophages and dendritic cells which trap soluble antigens and serve both innate and adaptive immune responses. Once the pDCs detect viral RNA or DNA, they pass through ordinary activation program to subsequently start secreting IFN $\alpha$. Conduits represent an important system of distribution channels for small soluble antigens and immune modulators (with molecular weight below $70 \mathrm{kDa}$ ). They extend from subcapsular sinus floor through the T-cell zone and form a contiguous lumen with fluid channels around the high endothelial venules (HEVs), thus making a network highly connected with the cortex capillaries and venules [12, 27]. The bulk flow of water and tracers passing via conduits is documented [16]. As the IFN $\alpha$ molecules mass is rather low, $\sim 17 \mathrm{kDa}$, they could also enter the conduit system. The paradigmatic lymph

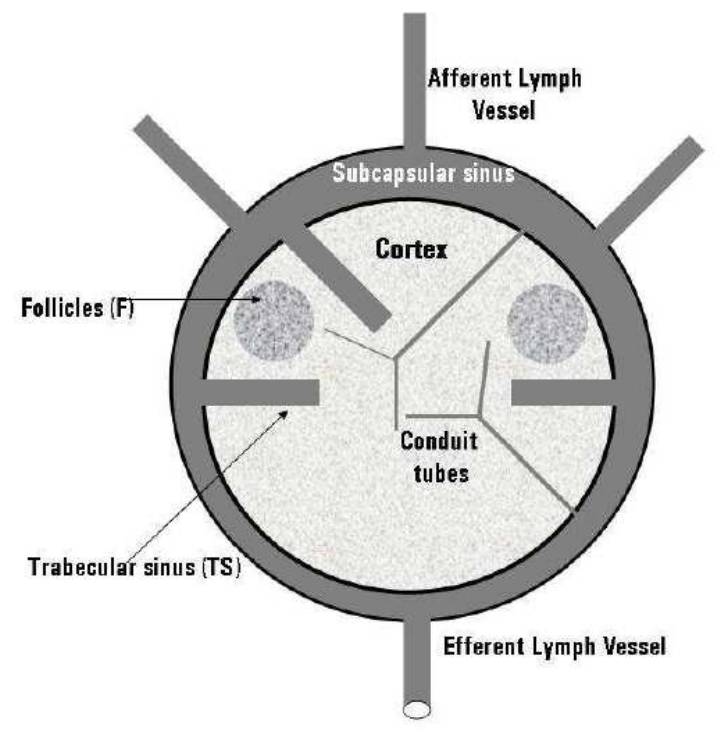

Figure 2: Schematic representation of a paradigmatic secondary lymphoid organ. SLO major functional building blocks are subcapsular sinus, trabecular sinus, conduits, B-cell follicles, T-cell zone.

node domain $\Omega$ is schematically described in Fig. 2 using a constructive solid geometry representation by combining three subdomains $\Omega_{i}, i=1,2,3$. Each subdomain is made of composition 
of geometric primitives, such as spheres and cylinders differing in their sizes and orientation. The Open CASCADE technology (see http://www.opencascade.org) was used to construct 3D geometric model of a paradigmatic lymph node. The first domain $\Omega_{1}$ is topologically defined as follows: $\left(S_{\text {out }} \backslash S_{\text {inn }}\right) \cup C_{T S} \cup C_{C T}$, where $S_{\text {out }}$ and $S_{\text {inn }}$ are the outer and inner spheres of diameters 2 $\mathrm{mm}$ and $1.9 \mathrm{~mm}$, respectively; $C_{T S}=\bigcup_{i=1}^{4} C_{i}$ stands for the union of 4 right circular cylinders with $0.05 \mathrm{~mm}$ diameter and $0.5 \mathrm{~mm}$ length; and $C_{C T}=\bigcup_{i=1}^{2} Y_{i}$ is a set of two conduits having $Y$-shape and $0.0005 \mathrm{~mm}$ diameter. The second domain $\Omega_{2}$, representing the B-cell follicles, is a union of 4 disconnected spheres $\bigcup_{i=1}^{4} S_{F, i}$ with a diameter of $0.2 \mathrm{~mm}$ located inside $S_{\text {inn. }}$. Finally, the third domain $\Omega_{3}$ topologically can be described as $S_{\text {inn }} \backslash\left(\Omega_{2} \cup C_{T S} \cup C_{C T}\right)$. The final Open CASCADE-based 3D geometric model of the lymph node consists of 50 vertices, 62 curved edges and 30 curved faces. The solid geometry model and its mesh approximation are presented in Fig. 3. This geometry requires multiple length scales resolution for the representation of the conduits and overall major domains. To this end we used the mesh approximation based on unstructured-mesh approach. The CAD designed geometric model was imported into the Ani3D
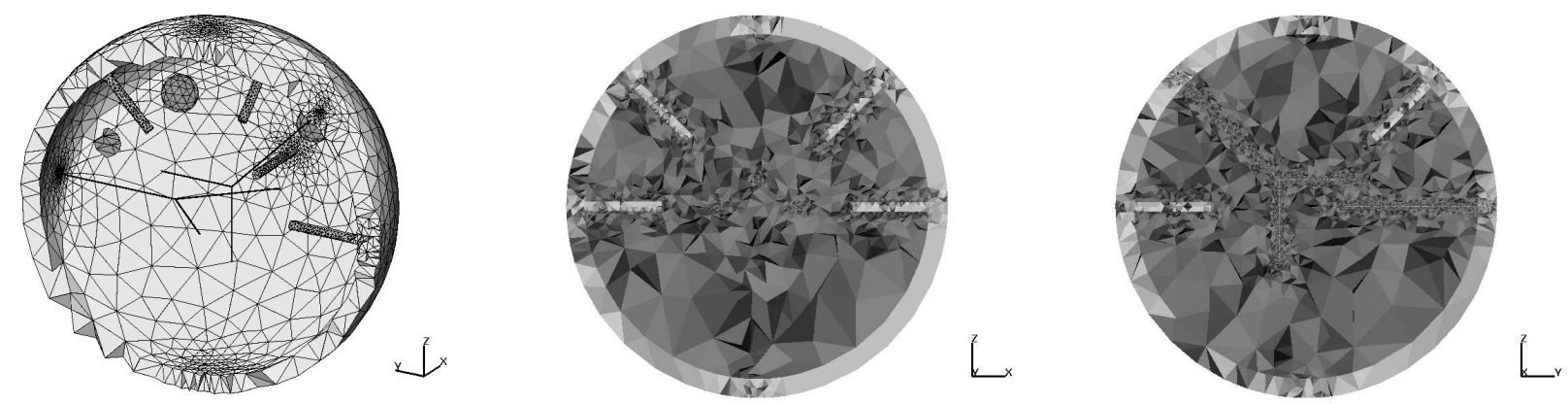

Figure 3: Reconstruction of the 3D geometry of the secondary lymphoid organ. Slice planes showing the tetrahedral spatial discretization of the SLO: left: slice plane for the subdomain $\Omega_{1}$; middle: slice plane $x z$; right: slice plane $y z$. Refining of mesh approximating conduits is an important feature of the meshing algorithm. The domain $\Omega_{1}$ is shown in yellow (or light grey), the follicles domain $\Omega_{2}$, consisting of small spheres, in green (or dark grey), and the subdomain $\Omega_{3}$ in grey.

mesh generation toolkit. Ani3D is a solid modeler-based preprocessing tool for robust generation of three-dimensional unstructured tetrahedral meshes [8]. Ani3D algorithms control and automate much of the meshing process. The tetrahedrization technology implements advancing front methods supplemented by Delaunay meshing technique to deal with void faces. Notice, that the mesh is a fine-grained closer to conduits in order to properly represent the structure of conduits, whose diameter is about three orders of magnitude smaller than the lymph node one. The lymph node surface approximating triangle mesh consists of 24720 vertices and 95732 triangles. The generated tetrahedral mesh model of the lymph node containing 103891 vertices and 619691 tetrahedrons was further improved using mesh smoothing implemented in aniMBA toolkit of Ani3D package. The resulting mesh is characterized by the minimal cell quality of $q_{\min }=0.08$ and the total number of tetrahedra is reduced to 594898 . 
Experimental studies reviewed in [16] indicate that a small molecular tracer was quickly distributed from the subcapsular sinus into the B-cell follicle, where it located diffusely between the lymphocytes. In contrast, within the T-cell zone the tracer distribution was restricted to conduits. It has been proposed that the differences in the distribution results from the biophysical characteristics of the T-cell zone, in particular, a hydraulic conductivity. Although direct measurements of the hydraulic conductivity within the T-cell area are missing, it is likely that both diffusion and convection are extremely low. The B-cell zone is considered to have a larger hydraulic conductivity that the T-cell zone. The above semi-quantitative observations have direct implications for the diffusion properties of soluble molecules like interferon. We assumed that the diffusion in domains $\Omega_{1}$ to $\Omega_{3}$ is characterized by the rates obeying the following diffusion coefficients ranking: $D_{1} \gg D_{2} \gg D_{3}$. As the molecular weight of IFN $\alpha$ is very close to that of myoglobin, we used the following estimate of the diffusion coefficient $D^{*}=0.16 \mathrm{~mm}^{2} / \mathrm{h} \mathrm{[13]} \mathrm{as} \mathrm{a} \mathrm{baseline} \mathrm{value.}$

\section{Numerical results}

The reaction-diffusion partial differential equation governing the interferon dynamics was used to analyze the steady-state distribution of IFN $\alpha$ across the subdomains of the 3D lymph node geometry. The corresponding reduced model (second order elliptic equation) reads:

$$
-\mathbb{D}\left(\frac{\partial^{2}}{\partial x^{2}}+\frac{\partial^{2}}{\partial y^{2}}+\frac{\partial^{2}}{\partial z^{2}}\right) I(\mathbf{x}, t)+d_{I} I(\mathbf{x}, t)=F(\mathbf{x}),
$$

where

$$
\mathbb{D}=D_{i} \cdot \mathbb{I} \text { in } \mathbf{x} \in \Omega_{i}, i=1, \ldots, 3, \quad F(\mathbf{x})=\sum_{k=1}^{K} \rho \delta\left(\mathbf{x}-\mathbf{x}_{k}\right) .
$$

The equation is understood in the weak sense. It considers interferon diffusion in the lymph node (the first term on the left side), its production and loss due to degradation and uptake by various cells (the first and the second terms on the right side, respectively). As the major IFN producers are $\mathrm{pDCs}$, we neglected the contribution of macrophages to the source term.

We considered the scenario, where the individual pDCs are located randomly, mainly in domain $\Omega_{1}$ around the polar region of the subcapsular sinus, although there was a no-zero probability of them to be placed in the upper half of the cortex and follicles. The algorithm for specifying the individual pDCs position with respect to the lymph node center in cylindrical coordinates, $\mathbf{x}_{k}=\left(z_{k}, r_{k}, \theta_{k}\right), k=1, \ldots, K$, makes use of random numbers $\left(\varepsilon_{i} \sim \mathcal{U}(0,1)\right)$ generated from a standard continuous uniform distribution as follows: $z_{k}=R_{L N}\left(1-\varepsilon_{1} \varepsilon_{2} \varepsilon_{3}\right), r_{k}=R_{z_{k}}(1-$ $\left.\varepsilon_{4} \varepsilon_{5} \varepsilon_{6}\right), \theta_{k}=2 \pi \varepsilon_{7}$, where $R_{L N}$ is the radius of the outer sphere approximating the LN, and $R_{z_{k}}=\sqrt{\left(R_{L N}^{2}-z_{k}^{2}\right)}$.

The boundary conditions were specified as follows. At the outer boundary of the domain $\Omega_{1}$ and the part of its inner boundary, overall defined as $\partial^{*} \Omega_{1}=\partial S_{\text {out }} \cup \partial S_{\text {inn }} \backslash\left(S_{\text {inn }} \cap C_{T S}\right) \backslash\left(S_{\text {inn }} \cap C_{C T}\right)$ 
the homogeneous Neumann boundary condition was used

$$
\mathbf{n} \cdot \mathbb{D} \nabla I(\mathbf{x}, t)=0 \text { on } \partial^{*} \Omega_{1} \text {. }
$$

The vector fields are not differentiable with respect to spatial coordinates at the other interfaces between the lymph node domains with different diffusion properties. Therefore, we impose the boundary conditions, which describe the continuity of both the IFN $\alpha$ concentration and the diffusion flux. If $\partial_{123} \Omega$ denotes the interface between the domains $\Omega_{2}$ and $\Omega_{3}, \Omega_{1}$ and $\Omega_{3}$, except for the part $\partial S_{\mathrm{inn}} \backslash\left(S_{\mathrm{inn}} \cap C_{T S}\right) \backslash\left(S_{\mathrm{inn}} \cap C_{C T}\right)$, then we require that

$$
I(\mathbf{x}, t) \text { continuous across } \partial_{123} \Omega, \quad \mathbf{n} \cdot \mathbb{D} \nabla I(\mathbf{x}, t) \text { continuous across } \partial_{123} \Omega \text {. }
$$

The numerical simulations of the full three-dimensional model were carried out using a monotone nonlinear cell-centered finite volume method developed for diffusion equations on conformal polyhedral meshes in [9]. The practically observed convergence order was about two. The computations were performed on Pentium IV PC with the software written in C. The diffusion coefficients in subdomains 1 to 3 we set as follows: $D_{1}=D^{*}$ (see Section 4), $D_{2}=0.1 D^{*}$ and $D_{3}=0.01 D^{*}$.
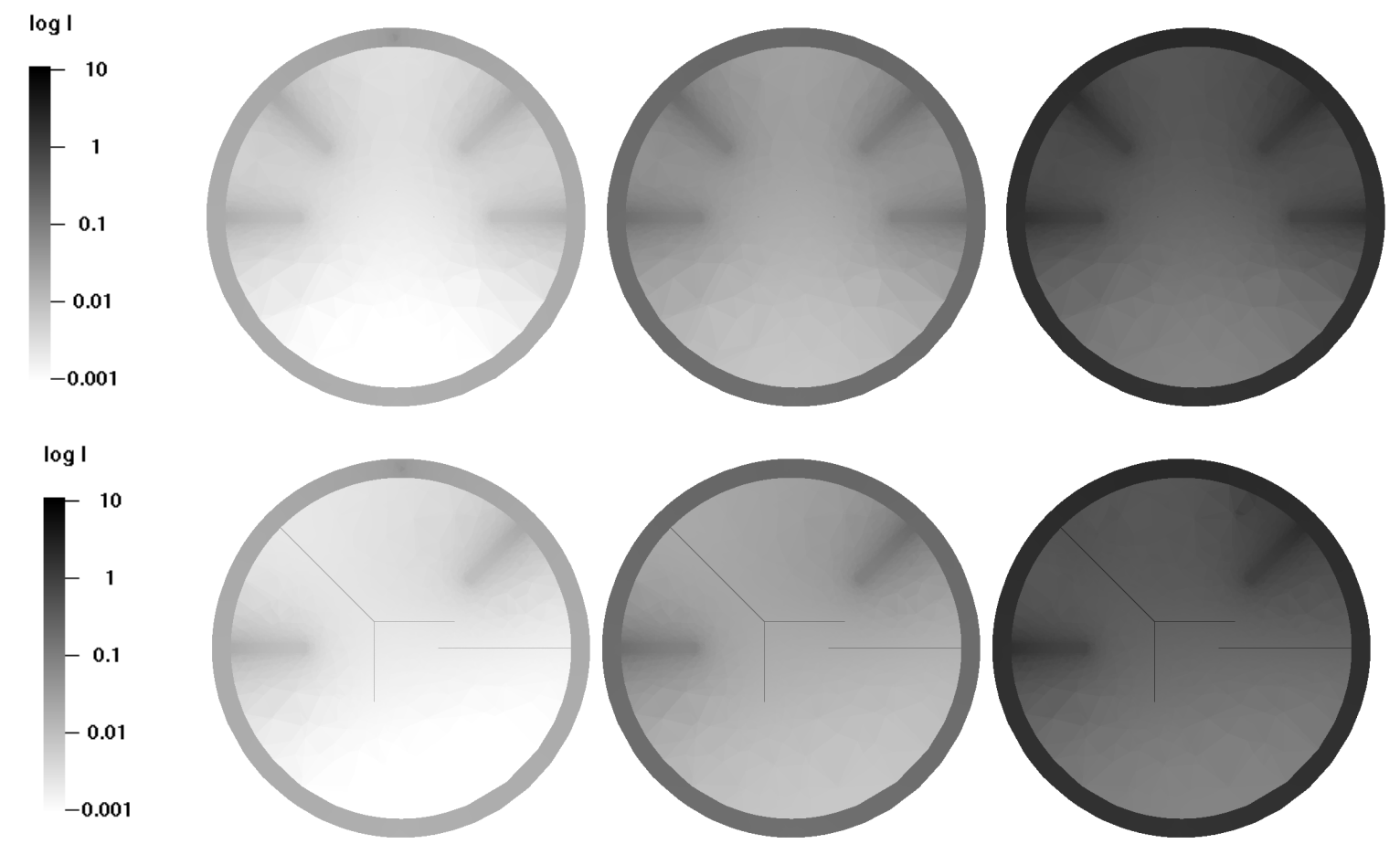

Figure 4: Simulated stationary distribution of IFN $\alpha$ across the paradigmatic lymph node for various number of source cells, pDCs, located randomly, mainly in the subcapsular sinus around the upper pole. Upper panel: OXZ cross-section; left: 1 pDC, middle: 10 pDCs, right: 100 pDCs. Lower panel: OYZ cross-section; left: $1 \mathrm{pDC}$, middle: $10 \mathrm{pDCs}$, right: $100 \mathrm{pDCs}$. The logarithmic color bars represent the concentration of the interferon $\left(\mathrm{pg} / \mathrm{mm}^{3}\right)$. 
We started by studying the interferon spatial distribution in the LN for different number of activated plasmacytoid dendritic cells. Figure 4 shows the stationary IFN $\alpha$ distribution across the LN for the source size (parameter $M$ in equation (5.1)) consisting of 1,10 and $100 \mathrm{pDCs}$. These numbers correspond to the fraction of interferon secreting cells covering the range of about $0.15 \%$ to $15 \%$ of the total pDCs in the lymph node. The IFN $\alpha$ distribution appears to be highly inhomogeneous with the differences in concentration ranging over two orders of magnitude between different regions. The subdomain $\Omega_{1}$ (subcapsular sinus, trabecular sinuses and conduits) is characterized by much higher concentration of the interferon- $\alpha$ than subdomain $\Omega_{3}$ representing T-cell zone. ApDCs number increase from 1 to 100 results in the rise of the concentration field of IFN $\alpha$ so that larger parts of the $\mathrm{LN}$ become protected against virus infection. In Fig. 5 there are shown the subdomains, where the IFN $\alpha$ concentration is below the threshold values of $0.01,0.1$ and 1 $\mathrm{pg} / \mathrm{mm}^{3}$, for the corresponding numbers of pDCs. The results suggest that as few as $10 \mathrm{pDCs}$ in the lymph node secrete enough interferon to ensure the protection of APCs (such as macrophages) in the whole subdomain $\Omega_{1}$.
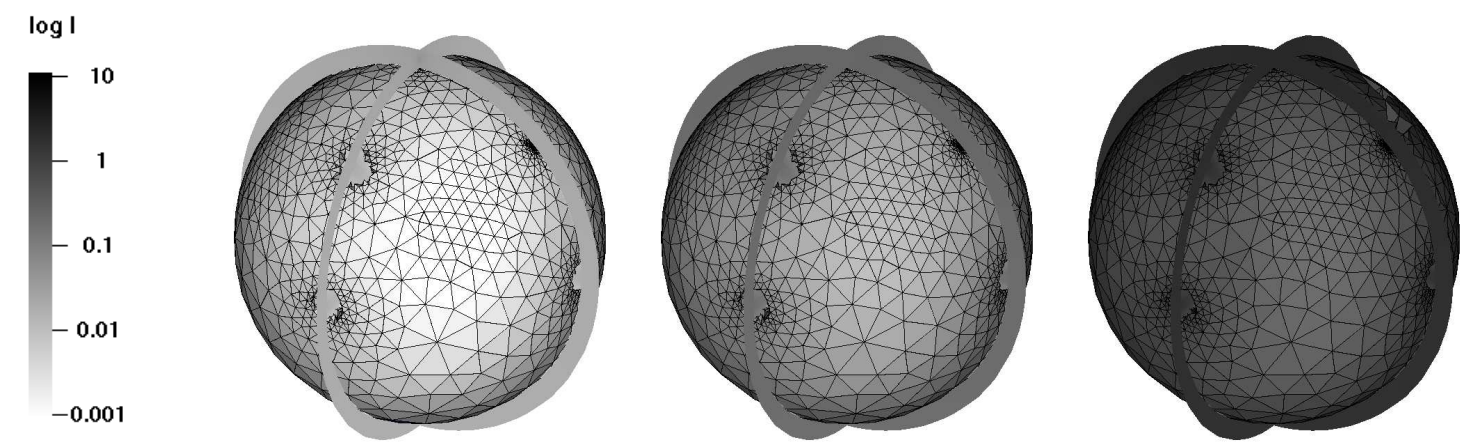

Figure 5: Simulated stationary distribution of IFN $\alpha$ across the paradigmatic lymph node for various number of source cells, pDCs, located randomly, mainly in the subcapsular sinus around the upper pole, with the tetrahedra showing the regions in which the IFN concentration is below some threshold $\theta$. OXZ and OYZ cross-sections are shown. Left: $1 \mathrm{pDC}$ with $\theta=0.01 \mathrm{pg} / \mathrm{mm}^{3}$, middle: $10 \mathrm{pDCs}$ with $\theta=0.1 \mathrm{pg} / \mathrm{mm}^{3}$, right: $100 \mathrm{pDCs}$ with $\theta=1.0 \mathrm{pg} / \mathrm{mm}^{3}$. The logarithmic color bar represents the concentration of the interferon $\left(\mathrm{pg} / \mathrm{mm}^{3}\right)$.

Then a sensitivity of the stationary interferon concentration to the rate of loss $d_{I}$ has been studied. The simulation results presented in Fig. 6 show that 10-fold increase leads to about 2fold decrease of the maximum concentration in subcapsular domain and conduits but dramatically reduces the amount of available interferon in the B- and T-cell zones.

\section{Concluding remarks}

This study results suggest that the spatial stationary distribution of IFN $\alpha$ is essentially heterogeneous across the lymph node. Highly protected subdomains such as sinuses, conduits, co-exist 

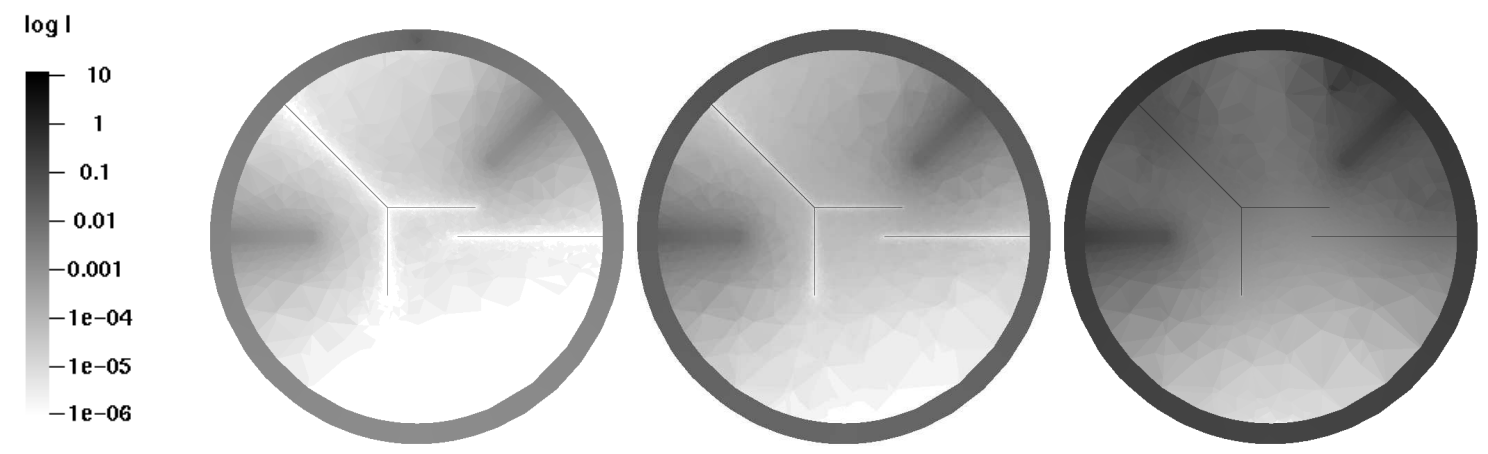

Figure 6: Simulated stationary distribution of IFN $\alpha$ across the paradigmatic lymph node for various number of source cells, pDCs, located randomly, mainly in the subcapsular sinus around the upper pole with the interferon loss rate $d_{I}$ increased by 10-fold. OYZ cross-section is shown. Left: 1 pDC, middle: 10 pDCs, right: 100 pDCs. The logarithmic color bar represents the concentration of the interferon $\left(\mathrm{pg} / \mathrm{mm}^{3}\right)$.

with the regions, where the stationary concentration of IFN $\alpha$ is lower by about 100 -fold. This implies that for some infections the pathogens can escape the IFN $\alpha$ effect if the infected target cell are localized/migrate into poorly protected SLO regions. As the conclusions heavily depend on the assumed variations in hydraulic conductivity of various zones of the secondary lymphoid organs, further experimental analysis of the SLO compartments diffusion characteristics is required.

A fundamental issue in understanding the viral infections pathogenesis is a disruption of the lymphoid organs microanatomy studied experimentally in mice using LCMV system [26]. It was shown to have a profound effect on the immune responsiveness of the host. Similar problem exists for HIV infection where the collapse of the lymph node architecture is observed in humans progressing to AIDS (see [17]). An integrative approach to understanding HIV pathogenesis [11] requires appropriate mathematical tools to consider the spatial organization of the immune responses and homeostasis.

Although the reaction-diffusion PDEs are commonly used in mathematical biology and chemical kinetics, to our knowledge this is the first study where the soluble immune factors spatial distribution in secondary lymphoid organs is modelled for a realistic three-dimensional geometry of the lymph node. The approach can be readily extended to other immune system organs. Next important step will be to use the 3D geometry model to explore the spatio-temporal dynamics of IFN $\alpha$ and other soluble factors using convection-diffusion-reaction systems.

To set up the 3D modelling platform a complexity of the system was simplified by focusing on a steady-state distribution of the interferon and ignoring an influence of other sinks in SLO such as efferent lymph vessels, high endothelial venules. Still, the corresponding scenario is biologically instructive for some chronic infections characterized by a stationary level of pDCs activation.

A transfer of the powerful 3D modelling technologies to experimental studies dealing with the spatial organization and functioning of the immune system requires further major efforts of applied mathematicians and immunologists. The focus should be made on the development of 
hybrid multiphysics models which integrate the spatial migration of cells and soluble substances in the lymphoid system with the real 3D geometry of immune responses microanatomy in SLO, calibrated using high-resolution fluorescence microscopy data. Although, there is a growing interest to explore the spatial complexities of the immune processes using mathematical models $[2,3,4,14,18,25]$, the real 3D geometry of the SLO remains to be properly considered. Further studies of the similar to the presented here and recent imaging technology-driven computational developments [15] should provide a proper basis for a transition in the current modelling paradigm of mathematical immunology from a phenomenological, spatially degenerate approach to a multiphysics-based high-resolution description of immune responses in physiological environment of the organized lymphoid tissues [30].

\section{Acknowledgements}

The authors thank the anonymous reviewer for the insightful comments and suggestions. The authors are grateful to the Russian Foundation for Basic Research (08-01-00141, 10-01-91055, 11 01-00117), the program of the Russian Academy of Sciences "Basic Research for Medicine" and the joint French-Russian Program International de Cooperation Scientifique (PICS) "Mathematical modelling of blood diseases".

\section{References}

[1] S. Andrew, C.T.H. Baker, G.A. Bocharov. Rival approaches to mathematical modelling in immunology. J. Comput. Appl. Math., 205 (2007), 669-686.

[2] V. Baldazzi, P. Paci, M. Bernaschi, F. Castiglione. Modeling lymphocyte homing and encounters in lymph nodes. BMC Bioinform., 10 (2009), doi:10.1186/1471-2105-10-387.

[3] C. Beauchemin, N.M. Dixit, A.S. Perelson. Characterizing T cell movement within lymph nodes in the absence of antigen. J. Immunol., 178 (2007), 5505-5512.

[4] J.B. Beltman, A.F. Maree, J.N. Lynch, M.J. Miller, R.J. de Boer. Lymph node topology dictates T cell migration behavior. J. Exp. Med., 204 (2007), 771-780.

[5] G.A. Bocharov, G.I. Marchuk. Applied problems of mathematical modelling in immunology. Comput. Math. Math. Phys., 40 (2000), 1905-1920.

[6] G. Bocharov. Understanding complex regulatory systems: Integrating molecular biology and systems analysis. Transf. Med. Hemoth., 32 (2005), No. 6, 304-321.

[7] G. Bocharov, R. Zust, L. Cervantes-Barragan, T. Luzyanina, E. Chiglintcev, V.A. Chereshnev, V. Thiel, B. Ludewig. A systems immunology approach to plasmacytoid dendritic cell function in cytopathic virus infections. PLoS Pathogens, 6(7) (2010), e1001017.doi:10.1371/journal.ppat.1001017, 1-14. 
[8] A.A. Danilov. Unstructured tetrahedral mesh generation technology. Comput. Math. Math. Phys., 50 (2010), 146-163.

[9] A.A. Danilov, Yu.V. Vassilevski. A monotone nonlinear finite volume method for diffusion equations on conformal polyhedral meshes. Russ. J. Numer. Anal. Math. Modelling, 24 (2009), 207-227.

[10] Z. Faroogi, R.R. Mohler. Distribution models of recirculating lymphocytes. IEEE Trans. Biomed. Engrg., 36 (1989), 355-362.

[11] Z. Grossman, M. Meier-Schellersheim, W.E. Paul, L.J. Picker. Pathogenesis of HIV infection: what the virus spares is as important as what it destroys. Nat. Med., 12 (2006), 289-295.

[12] T. Junt, E. Scandella, B. Ludewig. Form follows function: lymphoid tissues microarchitecture in antimicrobial immune defense. Nature Rev. Immunol., 8 (2008), 764-775.

[13] J. Keener, J. Sneyd. Mathematical physiology. Springer-Verlag, New York, 1998.

[14] T.B. Kepler, C. Chan. Spatiotemporal programming of a simple inflammatory process. Immunol. Reviews, 216 (2007), 153-163.

[15] F. Klauschen, M. Ishii, H. Qi, M. Bajenoff, J.G. Egen, R.N. Germain, M. MeierSchellersheim. Quantifying cellular interaction dynamics in 3D fluorescence microscopy data. Nat. Protoc., 4 (2009), 1305-1311.

[16] T. Lammermann, M. Sixt. The microanatomy of $T$ cell responses. Immunol. Reviews, 221 (2008), 26-43.

[17] P. Lane, R.-P. Sekaly. HIV and the architecture of immune responses. Semin. Immunol. 20 (2008), 157-158.

[18] J.J. Linderman, T. Riggs, M. Pande, M. Miller, S. Marino, D.E. Kirschner. Characterizing the dynamics of CD4+ T cell priming within a lymph node. J. Immunol., 184 (2010), 2873-2885.

[19] G.I. Marchuk. Mathematical modelling of immune response in infectious diseases. Kluwer Academic Publishres, Dordrecht, 1997.

[20] G.I. Marchuk. Methods of Numerical Mathematics. Springer-Verlag, New York, 1982.

[21] G.I. Marchuk, V. Shutyaev, G. Bocharov Adjoint equations and analysis of complex systems: application to virus infection modeling. J. Comput. Appl. Math., 184 (2005), 177-204.

[22] R.R. Mohler, Z. Faroogi, T. Heilig. Lymphocyte distribution and lymphatic dynamics. In: Vistas in Applied Mathematics: Numerical Analysis, Atmospheric Sciences, Immunology. (Eds. A.V. Balakrishnan, A.A. Dorodnitsyn, and J.-L. Lions) 1986, 317333. 
[23] J.H. Meyers, J.S. Justement, C.W. Hallahan, E.T. Blair, Y.A. Sun, M.A. O'Shea, G. Roby, S. Kottilil, S. Moir, C.M. Kovacs, T.W. Chun, A.S. Fauci. Impact of HIV on cell survival and antiviral activity of plasmacytoid dendritic cells. PLoS ONE, 2 (2008), No. 5, e458. doi:10.1371/journal.pone.0000458

[24] R.R. Mohler, C. Bruni, A. Gandolfi. A systems approach to immunology. Proceedings of the IEEE, 68 (1980), 964-990

[25] A.S. Perelson, F.W. Wiegel. Scaling aspects of lymphocyte trafficking. J. Theor. Biol., 257 (2009), 9-16.

[26] E. Scandella, B. Bolinger, E. Lattmann, S. Miller, S. Favre, D.R. Littman, D. Finke, S.A. Luther, T. Junt, B. Ludewig. Restoration of lymphoid organ integrity through the interaction of lymphoid tissue-inducer cells with stroma of the T cell zone. Nature Immunol., 9 (2008), 667-675.

[27] F. Pfeiffer, V. Kumar, S. Butz, D. Vestweber, B.A. Imhof, J.V. Stein, B. Engelhardt. Distinct molecular composition of blood and lymphatic vascular endothelial cell junctions establishes specific functional barriers within the peripheral lymph node. Eur. J. Immunol., 38 (2008), 2142-2155.

[28] D.J. Stekel, C.E. Parker, M.A. Nowak. A model of lymphocyte recirculation. Immunol. Today, 18 (1997), No. 5, 216-21.

[29] D.J. Stekel. The simulation of density-dependent effects in the recirculation of T lymphocytes. Scand. J. Immunol., 47 (1998), 426-430.

[30] S. Stoll, J. Delon, T.M. Brotz, R.N. Germain. Dynamic imaging of T cell-dendritic cell interactions in lymph nodes. Science, 296 (2002), 1873-1876. 